\title{
Black hole entropy from loop quantum gravity in higher dimensions
}

\author{
N. Bodendorfer* \\ Institute for Gravitation and the Cosmos \& Physics Department, \\ Penn State, University Park, PA 16802, U.S.A.
}

(Dated: July 19, 2013)

\begin{abstract}
We propose a derivation for computing black hole entropy for spherical non-rotating isolated horizons from loop quantum gravity in four and higher dimensions. The state counting problem effectively reduces to the well studied 3+1-dimensional one based on an SU(2)-Chern-Simons theory, differing only in the precise form of the area spectrum.
\end{abstract}

PACS numbers: 04.50.Gh, 04.60.-m, 04.70.Dy

\section{INTRODUCTION}

The calculation of black hole entropy is one of the main tests for candidate theories of quantum gravity. Different gravitational theories, horizon topologies and higher dimensions offer a great variety of non-trivial tests for such candidate theories. Despite this plethora of examples, the resulting entropies, calculated by different approaches, are very similar. At the classical level, this is explained by the Wald formula [1]. Carlip [2] and Solodukhin [3] noticed that one can derive black hole entropy in any dimension using conformal field theory techniques. This derivation also results in the universal logarithmic corrections $-3 / 2 \log (A)$ [4, 5]. Still, it would be desirable to have a derivation from a fundamental quantum theory of gravity.

Loop quantum gravity (LQG) [6, 7] has matured into a serious candidate for such a theory. Here, the calculation of black hole entropy is translated to the calculation of the entropy of isolated horizons, see [8] for a review. The advantage of this viewpoint is that isolated horizons provide local definitions of the relevant horizons (e.g. black hole or cosmological) and an explicit knowledge of their complete past and future is not required.

The main non-trivial point which allows to compute the entropy of black holes within LQG is that the connection variables used induce new degrees of freedom which are associated to the isolated horizon and not the to bulk. The classical part of this derivation was extended to higher dimensions in [9] and to LanczosLovelock gravity in [10], where the recently introduced connection variables for higher-dimensional general relativity 11 14 were employed. While the horizon degrees of freedom can be rewritten in a form similar to a higherdimensional Chern-Simons theory, it turns out to be more economical to use a canonically conjugate pair of normals $n^{I}$ and $\tilde{s}^{I}$ as horizon degrees of freedom [9]. In this paper, we will use the latter formulation in order to generalize the entropy calculation to higher dimensions. It turns out that the problem of computing the entropy reduces largely to the one familiar from $3+1$ dimensions due to the fact that the horizon Hilbert spaces in different dimensions can be mapped 1 to 1 onto each other.

\section{ISOLATED HORIZON DEGREES OF FREEDOM}

In this section, we will recall the isolated horizon degrees of freedom using the dimension-independent connection variables as derived in [9, 10]. We will neglect the detailed treatment of spatial infinity in this paper, as it is not relevant for the discussion, see e.g. [15]. The main result is that one can perform a phase space extension from the Lorentzian ADM phase space [16] in $D+1$ spacetime dimensions to a canonical pair consisting of an $\mathrm{SO}(D+1)$ connection $A_{a I J}$ and its conjugate momentum $\pi^{a I J}$. Here, $a, b=1, \ldots, D$ are tensor indices on a spatial slice $\Sigma$ and $I, J=0, \ldots, D$ are internal $\operatorname{SO}(D+1)$ indices. Let $q_{a b}$ be the induced metric on $\Sigma$ and $K_{a b}$ the extrinsic curvature, which together parametrize the ADM phase space. We introduce the $(D+1)$-bein $e_{a}^{I}$ satisfying $q_{a b}=e_{a}^{I} e_{b}^{J} \delta_{I J}$ and the hybrid spin connection $\Gamma_{a I J}^{\mathrm{H}}$ annihilating it [17]. The information of $q_{a b}$ and $K_{a b}$ is contained in the connection variables as

$$
\begin{aligned}
\left(A_{a I J}-\Gamma_{a I J}^{\mathrm{H}}\right) \pi^{b I J} & \approx 2 \sqrt{q} q^{b c} K_{a c} \\
\pi^{a I J} & \approx 2 / \beta n^{[I} e_{b}^{J]} q^{a b} \sqrt{q},
\end{aligned}
$$

where $\beta \in \mathbb{R} \backslash\{0\}$ is a free constant. $\approx$ means equality on the constraint surface, defined by the $\mathrm{SO}(D+1)$ Gauß law and the simplicity constraint $\pi^{a[I J} \pi^{b \mid K L]}=0$. The simplicity constraint ensures that $\pi^{a I J} \approx 2 / \beta n^{[I} e_{b}^{J]} q^{a b} \sqrt{q}$, that is, it is the product of a normal $n^{I}$ and a densitized $D+1$-bein orthogonal to $n^{I}$, see [11, 12, 18] for further details, e.g. a topological sector in $3+1$ dimensions.

One can check that the Poisson brackets

$$
\left\{A_{a I J}(x), \pi^{b K L}(y)\right\}=\delta_{a}^{b} \delta_{I J}^{K L} \delta^{(D)}(x-y) \quad \text { on } \Sigma
$$

reproduce the ADM Poisson brackets on the constraint surface, thus confirming equivalence to the ADM formulation. The construction works analogously for $\mathrm{SO}(1, D)$ as gauge group, however the compactness of $\mathrm{SO}(D+1)$ is preferred for quantization purposes.

In the presence of a boundary $H$ of $\Sigma$ (here, the intersection of $\Sigma$ with an isolated horizon $\Delta$ ) with boundary unit normal $s^{a}$ pointing outward of $\Sigma$, the above calculation is only correct up to a boundary term, which results 
in the additional Poisson bracket

$$
\left\{\tilde{s}^{I}(x), n_{J}(y)\right\}=\beta \delta_{J}^{I} \delta^{(D-1)}(x-y) \quad \text { on } H
$$

with $\tilde{s}_{J}:=\sqrt{h} s^{a} e_{a J}$ and $h=\operatorname{det} h_{\alpha \beta}, \alpha, \beta=1, \ldots, D-1$ being the determinant of the induced metric $h_{\alpha \beta}$ on $H$ [22]. Classically, the product $2 n^{[I} \tilde{s}^{J]}$ is determined by continuity from the bulk fields as

$$
2 / \beta n^{[I} \tilde{s}^{J]}=\hat{s}_{a} \pi^{a I J}
$$

where $\hat{s}_{a}=s^{b} q_{a b} \sqrt{h / q}$ is an appropriately densitized conormal. (5) is the analogue of the isolated horizon boundary condition $F^{I J}(A) \propto e^{I} \wedge e^{J}$ familiar from the $3+1$ dimensional treatment 8]. In fact, (5) can be rewritten in the form $F^{I J}(A) \propto e^{I} \wedge e^{J}$ in $3+1$ dimensions [9].

Since $n^{I} \tilde{s}_{I}=0$ classically by construction, the information contained in these variables associated to the boundary is the $(D-1)$-area-density $\sqrt{h}=\sqrt{\tilde{s}^{I} \tilde{s}_{I}}$ of $H$. We consider the densitized bi-normals $L^{I J}:=2 / \beta n^{[I} \tilde{s}^{J]}$ since they already contain this information. Their Poisson algebra is given by

$$
\left\{L_{I J}(x), L_{K L}(y)\right\}=4 \delta^{(D-1)}(x-y) \delta_{L][I} L_{J][K}
$$

and thus by the Lie algebra $\operatorname{so}(D+1)$ at every point of $H$. We will base our quantization of the horizon degrees of freedom on this algebra.

A similar structure has been found for AshtekarBarbero variables in [19], where it was also emphasized that compatibility of (3), (5), and (6) leads us to use holonomy-flux variables, thus strengthening the uniqueness result [20, 21] of the Ashtekar-Isham-Lewandowski representation based on these variables even further.

\section{CONDITIONS ON $\Delta$}

We demand $\Delta$ to be a spherical non-rotating isolated horizon, see 9] for details. From this, it follows that the null normal $l^{\mu}, \mu=0, \ldots, D$, to the isolated horizon $\Delta$ satisfies $\nabla_{\alpha} l^{\mu}=\omega_{\alpha} l^{\mu}=0$, i.e. $l^{\mu}$ is covariantly constant on $H$. For the $\mathrm{SO}(D+1)$-based connection variables used in this paper, we find $\nabla_{\alpha} k^{I}=0$, with $k^{I}=\left(n^{I}+s^{I}\right) / \sqrt{2}$ and $s^{I}=s^{a} e_{a}^{I}$, where the connection used is the pullback of the (bulk) $\mathrm{SO}(D+1)$-connection on $\Sigma$ to $H$. Note that the internal vectors $k^{I}$ and $l^{I}=\left(n^{I}-s^{I}\right) / \sqrt{2}$ cannot be null, i.e. $k^{2}=l^{2}=1, l \cdot k=0$, and are thus not related to the spacetime null-vectors $l^{\mu}, k_{\mu}$ [23]. Since $n^{[I} \tilde{s}^{J]}=-k^{[I} \tilde{l}^{J]}$, we demand that the bi-normals $L^{I J}$ share a common vector, here $k^{I}$, to incorporate this classical property of an isolated horizon. We can cover $H$ with two contractible charts due to its spherical topology. On each of these charts, the gauge $k^{I}=$ const. is accessible. Moreover, $A_{\alpha}{ }^{I}{ }_{J} k^{J}=0$ in this gauge, so that there is a trivial identification of the $k^{I}(x)$ at different points of the two charts. While a more elaborate treatment might be desirable (see the discussion section), this leads us to demand the "off-diagonal" horizon simplicity constraints $L_{[I J}(x) L_{K L]}(y)=0$ for $x, y$ in the same chart, which should restrict us to the relevant degrees of freedom. We now need to investigate the constraint algebra.

\section{CONSTRAINT ALGEBRA}

The Gauß and spatial diffeomorphism constraints

$$
\begin{aligned}
G^{I J}\left[\Lambda_{I J}\right] & =-\int_{\Sigma} \pi^{a I J} D_{a} \Lambda_{I J}+\int_{H} \Lambda_{I J} L^{I J} \\
\mathcal{H}_{a}\left[N^{a}\right] & =\frac{1}{2} \int_{\Sigma} \pi^{a I J} \mathcal{L}_{N} A_{a I J}+\frac{1}{\beta} \int_{H} n^{I} \mathcal{L}_{N} \tilde{s}_{I}
\end{aligned}
$$

form a closing algebra, where $\mathcal{L}_{N}$ denotes the Lie derivative along the shift vector $N^{a}$, satisfying $N^{a} \hat{s}_{a}=0$ on $H$ to respect the boundary. Their associated vector fields are degenerate directions of the symplectic structure and they are consistent with (5). The same is true for the simplicity constraint and the Hamiltonian constraint, provided that the lapse function vanishes on $H$. Thus, the Hamiltonian constraint does not have to be taken into account for the horizon Hilbert space, as in [15]. Moreover, $A_{H}:=\int_{H} \sqrt{h} d^{D-1} x$ is a Dirac observable. As usual, see e.g [24], we assume that there exists at least one solution of the Hamiltonian constraint in the bulk which is compatible with a given configuration of punctures. Otherwise, a gauge fixing as in [25] could be employed. Up to here, the constraint algebra is closing (first class) [26].

The horizon simplicity constraints need a more thorough investigation. They do not form a closing algebra, see e.g. 13], and are thus second class in Dirac's terminology [27]. In order to be able to quantize them, we will select a maximally commuting subset adapted to the puncturing spin network in the next section, that is, we perform gauge unfixing [28]. This subset does however not form a closing algebra with the Gauß constraints, since the smearing functions $\Lambda_{I J}$ are not constant on $H$. We thus further restrict to constant $\Lambda_{I J}$ on $H$ and note that also in the $\mathrm{U}(1)$ framework in $3+1$ dimensions [15, 24], the restriction to constant $\Lambda_{I J}$ on $H$ becomes necessary, however for different reasons 7 ].

\section{QUANTIZATION}

While the $L_{I J}$ are classically determined by the bulk fields through continuity, i.e. by the boundary condition (5), this ceases to be the case in the quantum theory since the Hilbert space used is distributional by nature. Thus, in the same way as in $3+1$ dimensions, the $L_{I J}$ have to be promoted to independent degrees of freedom in the quantum theory. The complete Hilbert space is therefore a product of the bulk Hilbert space defined in [13] and the horizon Hilbert space, which is a product of $\mathrm{SO}(D+1)$ 
representation spaces, on which the Poisson algebra (6) of boundary degrees of freedom can be represented as the generators of the gauge group $\mathrm{SO}(D+1)$ 29].

The boundary condition (5) now has to be implemented as an operator. We construct fluxes by smearing $\hat{s}_{a} \pi^{a I J}$ over a $(D-1)$-surface tangent to $H$. The fluxes can be promoted to quantum operators in the usual way [13] and obey the same algebra as the $L_{I J}$, which confirms the consistency of the framework. The quantum analog of the boundary condition (5) is automatically solved due to this algebraic structure by contracting holonomies ending on $H$ with a respective representation index of the horizon Hilbert space. At points of $H$ where no spin network edge punctures, the boundary condition implies that the $\mathrm{SO}(D+1)$ representation is trivial. The non-trivial part of the horizon Hilbert space is thus generated at the finite number of points where the bulk spin network punctures $H$.

The quantum Gauß law is also automatically solved in this way on $H$. We refer to [7] for an explicit quantization of the first term in (77). Quantization of the second term cancels the first one. Still, comparison with the $3+1$ dimensional treatment 24] suggests that the horizon Hilbert space should be further restricted by a global gauge invariance condition, which is derived there by "shrinking a loop around the back of the sphere". This procedure is not readily available in higher dimensions. Instead, we follow the ideas of [30] and enforce global gauge invariance in the next section by tracing over gauge related bulk degrees of freedom. Alternatively, we could quantize Gauß-invariant spin networks directly, which would also result in $\mathrm{SO}(D+1)$ intertwiners as horizon states 31]. This procedure however has the technical problem that the simplicity constraint is not gauge invariant, but only covariant, and still needs to be implemented in the quantum theory.

The spatial diffeomorphism constraint selects diffeomorphism equivalence classes of spin networks, see [7] for details. The bulk simplicity constraint together with the boundary condition (5) restricts us to simple $\mathrm{SO}(D+1)$ representations on $H$, which are labelled by a single nonnegative integer $\lambda[13,18$. This is consistent with the fact that we could have imposed diagonal horizon simplicity constraints on the horizon degrees of freedom.

We still have to deal with the second class nature of the off-diagonal horizon simplicity constraints. For this, we choose a maximally commuting subset by imagining the edges puncturing $H$ in each of the two charts to be connected to a spin network vertex and following the recipe in [32]. It is easy to see that the subset is identical to a subset that would have been obtained from imposing the off-diagonal simplicity constraints on all of $H$ 33. Thus, for spherical topology, the maximal amount of offdiagonal simplicity constraints can be imposed. This further restricts the horizon state to a simple $\mathrm{SO}(D+1)$ intertwiner. Such an intertwiner has only simple repre- sentations in a given recoupling scheme as intermediate representations, see [32] for details.

Since there is a 1 to 1 correspondence of simple $\mathrm{SO}(D+1)$ intertwiners to $\mathrm{SU}(2)$ intertwiners by mapping the recoupling labels for a given recoupling scheme onto each other, the problem of finding the dimension of the horizon Hilbert space is reduced to the $3+1$-dimensional case 34]. The remaining step is to find an analogous restriction to a finite level of the Chern-Simons theory in $3+1$ dimensions. The finite value of the level translates into an upper bound on the spins puncturing the horizon and thus also on the ones in the recoupling scheme of the intertwiner. Such a bound can also be derived from considering that the area of the horizon slice $H$ is finite. The $(D-1)$-area operator $\hat{A}$ is constructed in the same

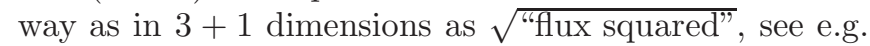
[13. for details. It operates diagonally on an edge labelled with a simple $\mathrm{SO}(D+1)$ representation. The eigenvalue is given by $8 \pi G \beta \sqrt{\lambda(\lambda+D-1)}, \lambda \in \mathbb{N}_{0}$. The total area of a horizon slice punctured by $P$ edges is thus

$$
A_{H}=8 \pi G \beta \sum_{i=1}^{P} \sqrt{\lambda_{i}\left(\lambda_{i}+D-1\right)} .
$$

It follows that the sum of the puncture labels, and accordingly also all the recoupling labels, are bounded by

$$
\sum_{i=1}^{P} \lambda_{i}<\sum_{i=1}^{P} \sqrt{\lambda_{i}\left(\lambda_{i}+D-1\right)}=\frac{A_{H}}{8 \pi G \beta} \leq k .
$$

for $k=\left\lceil A_{H} / 8 \pi G \beta\right\rceil$. The definition of $k$ is meant to resemble the level of the Chern-Simons theory in $3+1$ dimensions. Given this bound, the dimension of the intertwiner space at $H$ for given puncture labels $\lambda_{i}$ is [35]

$$
N\left(A_{H}, P, k\right)=\frac{2}{k+2} \sum_{d=1}^{k+1} \sin ^{2}\left(\frac{\pi d}{k+2}\right) \prod_{i=1}^{P} \frac{\sin \left(\frac{\pi d d_{i}}{k+2}\right)}{\sin \left(\frac{\pi d}{k+2}\right)},
$$

where $d_{i}=\lambda_{i}+1$, due to the 1 to 1 correspondence between $\mathrm{SU}(2)$ and simple $\mathrm{SO}(D+1)$ intertwiners. Note that this mapping is given by $\lambda=2 j[32]$.

\section{ENTROPY CALCULATION}

In order to compute the entropy of the isolated horizon, we first have to decide over which bulk degrees of freedom we want to trace. In any case, as mentioned in the previous section, we will trace over gauge related bulk states. This selects the $\mathrm{SO}(D+1)$-invariant subspace of the horizon Hilbert space due to the constancy of the smearing functions of the Gauß constraint, see also the discussion in [30, 36]. Thus, the relevant horizon states are gauge invariant by themselves. Up to this, there are two main proposals for tracing: the first one is to sum over all spin 
networks with fixed total horizon area $A_{H}$, thus having a variable number of punctures. This approach has been employed in the original calculation 24] and extended to the $\mathrm{SU}(2)$ approach [19] in [4, 37]. We can readily apply it and obtain an entropy proportional to $A_{H} / \beta$ at leading order. For $\lambda=1$ as the lowest possible representation label, we obtain the logarithmic correction $-3 / 2 \log (A)$ found by Carlip in any dimension [5] and thus confirm this calculation from an independent point of view.

On the other hand, one can argue that an observer outside of the black hole can in principle measure the spin network state, that is the graph (up to spatial diffeomorphisms) and the spin / intertwiner labels. Then, the number of punctures would be fixed. A more recent proposal along these lines involving an analytic continuation of the Barbero-Immirzi parameter $\gamma$ to the (anti)selfdual case $\pm i$ seems promising [38]. See [25, 39, 40] for more discussion on this route. The essential ingredients to this approach are to use a fixed number of punctures and the large spin limit. The method of [38] to analytically continue $\gamma$ in $3+1$ dimensions can be applied also in our dimension-independent treatment. In analogy to [38], we continue $\beta$ to $\pm i / 2$ and interpret $8 \pi G \sum_{i=1}^{P} j_{i}$ as the area, since $j \gg 1$ [41]. Since the dimension of the horizon Hilbert space for given puncturing spins is the same as in the 3+1-dimensional case, the results of 38. can be taken over verbatim, resulting in

$$
S=\log N\left(A_{H}, P, \mp i \beta k / 2\right)=\frac{A_{H}}{4 G}+\text { corrections. }
$$

\section{POLYHEDRAL VIEWPOINT}

A polyhedral viewpoint of the above calculation can be established in the following way: We recall that $\mathrm{SU}(2)$ intertwiners in the $3+1$-dimensional theory can be seen as the quantization of convex polyhedra in three dimensions 42]. Consider a polyhedron in $D$ spatial dimensions with $P(D-1)$-faces. By the Minkowski theorem [43], it is in 1 to 1 correspondence (up to translations) with a set of weighted normals $\tilde{s}_{n}^{i}=A_{n} s_{n}^{i}$ satisfying $\sum_{n} \tilde{s}_{n}^{i}=0$, where $A_{n}$ is the area of a the $n$-th polyhedral face and $s_{n}^{i}$ is a unit vector with $i=1, \ldots, D$. Let the polyhedron now live in a $D+1$ dimensional Euclidean space, that is we consider $\tilde{s}^{I}, I=0, \ldots, D$ and introduce an additional normal per face labelled $n_{n}^{I}$, so that $n_{n}^{I} \tilde{s}_{n}^{J} \delta_{I J}=0$. The natural symplectic structure is now given by $\left\{\tilde{s}_{m}^{I}, n_{n}^{J}\right\}=$ $\delta^{I J} \delta_{m n}$ and is similar to (4).

In order to still be able to apply the Minkowski reconstruction, we need to demand that $n_{n}^{I}=n^{I}$ for a fixed normal $n^{I}$. We thus introduce the bi-vectors $L_{n}^{I J}$ per face together with the diagonal simplicity constraints $L_{n}^{[I J} L_{n}^{K L]}=0$ and off-diagonal simplicity constraints $L_{n}^{[I J} L_{m}^{K L]}=0$. These constraints ensure that $L_{n}^{I J}=2 n^{[I} \tilde{s}_{n}^{J]}$ [18]. The symplectic structure of the bi- vectors is again similar to (6) and agrees with the one calculated from $n_{n}^{I}, \tilde{s}_{n}^{J}$ on the constraint surface of the simplicity constraints. We note the strong similarity to the symplectic structure in [42]. From the Minkowski reconstruction theorem, we have the additional constraint $\sum_{n} L_{n}^{I J}=0$.

As above, quantization of the Poisson-algebra of bivectors yields a Hilbert-space which is given by the product of individual $\mathrm{SO}(D+1)$-representations. The quantization of $\sum_{n} L_{n}^{I J}=0$ projects this Hilbert-space onto its $\mathrm{SO}(D+1)$-invariant part, while a maximally commuting subset of the simplicity constraints 32] yields a simple $\mathrm{SO}(D+1)$-intertwiner, which is in 1 to 1 correspondence with an SU(2)-intertwiner. Thus, the foundation of the ideas discussed in [42] extends to higher dimensions. A thorough understanding this viewpoint may be key in better understanding the 1 to 1 mappings of the simple intertwiner spaces in higher dimensions found in [32].

As was discussed in [44, 45] in the four-dimensional context, the interpretation of the horizon states as different shapes of the horizon, i.e. polyhedra, seems in conflict with symmetry assumption made in the classical calculation. However, this tension was resolved in [44, 45] by showing that the classical calculation can be extended to arbitrary isolated horizons without changing the algebraic structure of the horizon degrees of freedom, thus allowing for the same quantization. It would certainly be interesting to extend this viewpoint to higher dimensions. In other words, one would like to show that the results of [9, 10] also hold for generic, e.g. rotating, isolated horizons, without changing the algebraic structure of the horizon degrees of freedom and the boundary condition. We leave this problem for further research.

\section{COMMENTS}

Lanczos-Lovelock gravity, a generalization of higher-dimensional GR on the same phase space, has been discussed in 10] in the presence of an isolated horizon. The entropy calculation presented here is compatible with these results and the Wald entropy [1, 46] emerges at leading order (with correct prefactor for the analytic continuation $\beta \rightarrow \pm i / 2$ ). This is due to the fact that the area density $\sqrt{h}$ on $H$ is replaced by a Wald entropy density in the relevant calculations.

For connection variables on the boundary, e.g. the Chern-Simons symplectic structures derived in [9], one could construct a locally gauge invariant off-diagonal horizon simplicity constraint by parallel transporting both $L^{I J}$ to the same point. This way, local gauge invariance on $H$ might be kept. Though seemingly harder to achieve, a quantization along this route would be preferable. In contrast, the constraint used in this paper breaks local $\mathrm{SO}(D+1)$-invariance on $H$ explicitly by dropping these parallel transporters. 
The implementation of the simplicity constraints proposed in 32 and used in this paper faces two potential problems in general situations: The chosen maximal subset might not commute with the action of the Hamiltonian constraint and the choice of subset might have an influence on the physics, i.e. different choices could be unitarily inequivalent at the level of observables. For the proposed application however, both objections do not apply, since the lapse function vanishes on $H$ and the dimension of the simple intertwiner space does not depend on the chosen subset.

In $2+1$ dimensions, the simplicity constraints are automatically satisfied. If we still restrict to global gauge invariance on $H$ and apply the arguments of [30], the derivation goes through also here.

\section{ACKNOWLEDGEMENTS}

NB was supported by the NSF Grant PHY-1205388 and the Eberly research funds of The Pennsylvania State University. Discussions with Abhay Ashtekar, Marc Geiller, Alok Laddha and especially Yasha Neiman are gratefully acknowledged. Final improvements of this work were supported by a Feodor Lynen Research Fellowship of the Alexander von Humboldt-Foundation.

* norbert@gravity.psu.edu

[1] R. Wald, Physical Review D 48 (1993) R3427-R3431, arXiv:gr-qc/9307038

[2] S. Carlip, Physical Review Letters 82 (1999) 2828-2831, arXiv:hep-th/9812013.

[3] S. N. Solodukhin, Physics Letters B 454 (1999) 213, hep-th/9812056.

[4] R. Kaul and P. Majumdar, Physical Review Letters 84 (2000) 5255-5257, arXiv:gr-qc/0002040

[5] S. Carlip, Classical and Quantum Gravity 17 (2000) 4175-4186, arXiv:gr-qc/0005017.

[6] C. Rovelli, Quantum Gravity. Cambridge University Press, Cambridge, 2004.

[7] T. Thiemann, Modern Canonical Quantum General Relativity. Cambridge University Press, Cambridge, 2007.

[8] J. Diaz-Polo and D. Pranzetti, SIGMA 8 (2012) 048, arXiv:1112.0291 [gr-qc].

[9] N. Bodendorfer, T. Thiemann, and A. Thurn, arXiv:1304.2679 [gr-qc].

[10] N. Bodendorfer and Y. Neiman, arXiv: 1304.3025 [gr-qc]
N. Bodendorfer, T. Thiemann, and A. Thurn, Classical and Quantum Gravity 30 (2013) 045001, arXiv:1105.3703 [gr-qc].

[12] N. Bodendorfer, T. Thiemann, and A. Thurn, Classical and Quantum Gravity 30 (2013) 045002, arXiv:1105.3704 [gr-qc].

[13] N. Bodendorfer, T. Thiemann, and A. Thurn, Classical and Quantum Gravity 30 (2013) 045003, arXiv:1105.3705 [gr-qc].
[14] N. Bodendorfer, T. Thiemann, and A. Thurn, Classical and Quantum Gravity 30 (2013) 045004, arXiv:1105.3706 [gr-qc]

[15] A. Ashtekar, A. Corichi, and K. Krasnov, Advances in Theoretical and Mathematical Physics 3 (2000) 419-478, arXiv: gr-qc/9905089.

[16] R. Arnowitt, S. Deser, and C. W. Misner, in Gravitation: An introduction to current research ( L. Witten, ed.), (New York), pp. 227-265, Wiley, 1962. arXiv:gr-qc/0405109.

[17] P. Peldan, Classical and Quantum Gravity 11 (1994) 1087-1132, arXiv:gr-qc/9305011

[18] L. Freidel, K. Krasnov, and R. Puzio, Advances in Theoretical and Mathematical Physics 3 (1999) 1289-1324, arXiv:hep-th/9901069.

[19] J. Engle, K. Noui, A. Perez, and D. Pranzetti, Physical Review D 82 (2010) 044050, arXiv: 1006.0634 [gr-qc]

[20] J. Lewandowski, A. Okolów, H. Sahlmann, and T. Thiemann, Communications in Mathematical Physics $\mathbf{2 6 7}$ (2006) 703-733, arXiv:gr-qc/0504147.

[21] C. Fleischhack, Communications in Mathematical Physics 285 (2009) 67-140, arXiv:math-ph/0407006

[22] It should be noted that in order for $n^{I}$ to be a unit vector, the Poisson bracket should be modified slightly to ensure consistency with $n^{I} n_{I}=1$. This already follows from reconstructing this bracket from (6). We will comment on this detail in a future publication.

[23] For $\mathrm{SO}(1, D)$ as internal gauge group, we would have $\nabla_{\alpha} l^{I}=0$ with $l^{2}=0$.

[24] A. Ashtekar, J. Baez, and K. Krasnov, Advances in Theoretical and Mathematical Physics 4 (2000) 1-94, arXiv: gr-qc/0005126.

[25] N. Bodendorfer, A. Stottmeister, and A. Thurn, Classical and Quantum Gravity 30 (2013) 082001, arXiv:1203.6525 [gr-qc]

[26] We refrain from restricting the gauge invariance on $H$, as e.g. done in [15], since the action principle detailed in [9] does not require any such gauge fixing. This mimics as closely as possible the more physical case of spin networks extending inside the horizon [30].

[27] P. A. M. Dirac, Lectures on Quantum Mechanics. Belfer Graduate School of Science, Yeshiva University Press, New York, 1964.

[28] P. Mitra and R. Rajaraman, Physics Letters B 225 (1989) 267-271.

[29] More precisely, one would regularize the $L^{I J}$ like fluxes by smearing them over small surfaces on $H$.

[30] K. Krasnov and C. Rovelli, Classical and Quantum Gravity 26 (2009) 245009, arXiv:0905.4916 [gr-qc].

[31] We underline the discrepancy between solving the Gauß law at the classical and quantum level on $H$. In our context, we consider the classical solution to be the physical one, see also [30]. A similar discrepancy is familiar from the simplicity constraint.

[32] N. Bodendorfer, T. Thiemann, and A. Thurn, Classical and Quantum Gravity 30 (2013) 045005, arXiv:1105.3708 [gr-qc]

[33] To see this, divide the sphere into two charts. Choose a recouping scheme in which all the representations $\lambda_{1}, \ldots, \lambda_{m}$ from chart 1 are coupled to a single representation, which in turn is coupled to the representations on chart 2. Simplicity of the intermediate spin follows from $\left(L_{1}^{[I J}+\ldots+L_{m}^{[I J}\right)\left(L_{1}^{K L]}+\ldots+L_{m}^{K L]}\right)=0$, which 
is contained in both maximally commuting subsets.

[34] J. Engle, K. Noui, and A. Perez, Physical Review Letters 105 (2010) 031302, arXiv:0905.3168 [gr-qc]

[35] J. Engle, K. Noui, A. Perez, and D. Pranzetti, Journal of High Energy Physics 2011 (2011) 16, arXiv:1103.2723 [gr-qc]

[36] W. Donnelly, Vacuum entanglement and black hole entropy of gauge fields. PhD thesis, University of Maryland, 2012.

[37] R. K. Kaul and P. Majumdar, Physics Letters B 439 (1998) 267-270, arXiv:gr-qc/9801080.

[38] E. Frodden, M. Geiller, K. Noui, and A. Perez, arXiv: 1212.4060 [gr-qc]

[39] N. Bodendorfer and Y. Neiman, Classical and Quantum Gravity 30 (2013) 195018, arXiv:1303.4752 [gr-qc].

[40] D. Pranzetti, arXiv:1305.6714 [gr-qc]
[41] Note that the area spectrum for the SO(4)-based connection variables is double of that of the SU(2)-based variables, resulting from the fact that the representations in the $\mathrm{SO}(4)$ theory are restricted to $\lambda / 2=j_{+}=j_{-}$[13].

[42] E. Bianchi, P. Dona, and S. Speziale, Physical Review D 83 (2011) 044035, arXiv:1009.3402 [gr-qc].

[43] H. Minkowski, Nachr. Ges. Wiss. Gött., Math.-Phys. (1897) $198-220$.

[44] C. Beetle and J. Engle, "Generic isolated horizons in loop quantum gravity," Classical and Quantum Gravity $\mathbf{2 7}$ (2010) 235024, arXiv:1007.2768 [gr-qc].

[45] C. Beetle and J. Engle, "Entropy of generic quantum isolated horizons," Journal of Physics: Conference Series 360 (2012) 012037, arXiv:1112.3994 [gr-qc].

[46] T. Jacobson and R. Myers, Physical Review Letters 70 (1993) 3684-3687, arXiv:hep-th/9305016 